Relations industrielles

Industrial Relations

\title{
Career Management, by Marion S. Kellog, New York, American Management Association, 1972, 200 p.
}

\section{Jean-Pierre Beaulieu}

Volume 29, numéro 4, 1974

URI : https://id.erudit.org/iderudit/028575ar

DOI : https://doi.org/10.7202/028575ar

Aller au sommaire du numéro

Éditeur(s)

Département des relations industrielles de l'Université Laval

ISSN

0034-379X (imprimé)

1703-8138 (numérique)

Découvrir la revue

Citer ce compte rendu

Beaulieu, J.-P. (1974). Compte rendu de [Career Management, by Marion S.

Kellog, New York, American Management Association, 1972, 200 p.] Relations

industrielles / Industrial Relations, 29(4), 895-895.

https://doi.org/10.7202/028575ar

Tous droits réservés @ C Département des relations industrielles de l'Université Laval, 1974
Ce document est protégé par la loi sur le droit d'auteur. L’utilisation des services d'Érudit (y compris la reproduction) est assujettie à sa politique d'utilisation que vous pouvez consulter en ligne.

https://apropos.erudit.org/fr/usagers/politique-dutilisation/ 
à l'Alcan, aucun article ne relate une expérience vécue de D.O. dans le milieu de l'entreprise privée. C'est assez paradoxal lorsqu'on songe à la multiplicité et à la diversité des expériences de D.O. faites tant aux Etats-Unis qu'au Canada dont, au moins, quelques-unes dans la province de Québec.

\section{Laurent BELANGER}

\section{Université Laval}

Career Management, by Marion S. Kellog, New York, American Management Association, 1972, 200 p.

Le développement de la carrière des employés salariés (cadres, professionnels, ou équivalents) se présente sous un nouvel aspect depuis quelques années. Les règles du jeu ont été sensiblement modifiées. De moins en moins de corporations ont maintenant la réputation d'offrir une sécurité d'emploi à vie pour leurs employés; de plus en plus de jeunes diplômés universitaires accordent plus de loyauté à leur profession plutôt qu'à leur employeur initial.

Ces nouvelles règles de jeu sont à la base de l'ouvrage de Kellog. A partir de ce point de référence, l'auteur dégage les implications qui en résultent tant pour l'employé que pour l'employeur.

Aujourd'hui l'employé est le premier responsable de sa carrière. Il ne peut plus ou ne doit plus s'en remettre à la discrétion de son supérieur en espérant que les événements et un effort soutenu le favorisent. Il doit d'abord déterminer sa propre orientation, ses objectifs de carrière puis en faire part à l'organisation dont il fait partie et vérifier périodiquement si elle peut toujours satisfaire ses attentes.

De son côté, l'organisation doit soutenir et faciliter ces efforts de la part de ses membres. Distinguant fort judicieusement la planification de maind'œuvre de la planification de carrière, Kellog présente diverses politiques et pratiques administratives qui doivent conduire à l'intégration des deux dans un effort total de planification et de développement des ressources humaines.

Sans être nécessairement d'accord avec chacune de ces politiques et pratiques, dans l'ensemble nous y retrou- vons plusieurs éléments d'intérêt que nous apprécions.

Comme la majorité des ouvrages publiés par l'AMA, ce volume s'adresse davantage aux praticiens qu'aux théoriciens, les premiers devant se recruter cette fois non seulement chez les directeurs du personnel mais aussi parmi les administrateurs, ceux qui ont la responsabilité immédiate de personnel. Egalement les employés qui sont les premiers concernés par le sujet devraient s'intéresser à ce volume. Kellog présente plusieurs idées qui requièrent une nouvelle conception du développement de carrière tant pour les salariés que pour leurs supérieurs. C'est en quelque sorte le défi qui s'offre à tous ceux qui veulent d'une part un travail intéressant et motivant, un sentiment de réalisation de soi-même et de croissance, d'autre part un personnel qualifié, intéressé, et prêt à faire face à de nouvelles responsabilités pour éviter la routine et la facilité.

\section{Alcan, Arvida}

\section{Jean-Pierre BEAULIEU}

Equity and Efficiency Effects from Manpower Programs, by Corry F. Azzi, Toronto, D.C. Health and Co., 1973, $97 \mathrm{pp}$.

Fondamentalement, cet ouvrage traite d'un des types possibles de l'évaluation des programmes de main-d'œuvre, l'analyse coûts-bénéfices. Cependant, l'approche utilisée se veut différente comme le laisse voir cette citation du premier paragraphe du livre:

«Although the recent development and proliferation of federally subsidized manpower programs designed to benefit the disadvantaged has received considerable attention, studies which have attempted to quantify the costs and benefits of these programs have been inadequate $\gg$.

$\mathrm{La}$ base de ce reproche réside dans le fait que ces études ont traditionnellement supposé que les salaires égalaient la valeur du produit marginal, ce qui, selon Azzi, est un modèle très irréaliste du comportement de la firme.

Contrairement à ce que peut laisser croire son titre, ce livre ne traite pas des programmes de main-d'œuvre, mais plutôt exclusivement de programmes de formation professionnelle en cours 